\title{
Loss of Cancellous Bone Mass and Connectivity in Ovariectomized Rats Can Be Restored by Combined Treatment with Parathyroid Hormone and Estradiol
}

\author{
Victor Shen, * David W. Dempster, ** Richard Birchman, * Ruth Xu, * and Robert Lindsay *s \\ * Regional Bone Center, Helen Hayes Hospital, New York State Department of Health, W. Haverstraw, New York 10993; and \\ Departments of ${ }^{\ddagger}$ Pathology and ${ }^{\S}$ Medicine, Columbia University, New York 10032
}

\begin{abstract}
To evaluate the potential use of a combination of antiresorption and bone formation-promoting agents as a treatment for postmenopausal osteoporosis, we examined the effects of combined and separate administration of estrogen (17 $\beta$-estradiol, $30 \mu \mathrm{g} /$ kg per d, s.c.) and parathyroid hormone (rPTH [1-34], $40 \mu \mathrm{g} /$ kg per d, s.c.) on the proximal tibia of ovariectomized (Ovx) rats. The treatments lasted for 4 wk and were initiated 1,3 , and 5 wk after surgery. Ovx resulted in rapid loss of cancellous bone volume ( $\mathrm{Cn}-\mathrm{BV} / \mathrm{TV})$ as well as trabecular connectivity, as determined by two dimensional strut analysis. When administered in a preventive mode, treatment beginning 1 wk postOvx, estrogen or PTH treatment alone preserved Cn-BV/TV and trabecular connectivity, and combined estrogen and PTH treatment caused a $40 \%$ increment in Cn-BV / TV while maintaining comparable trabecular connectivity with that seen in the Sham-operated animals. When administered in a curative mode to rats with established osteoporosis, treatments beginning 3 or 5 wk post-Ovx, estrogen or PTH treatment alone prevented further loss of connectivity and Cn-BV/TV, whereas the combined treatment resulted in as much as a $300 \%$ improvement in one of the parameters of trabecular connectivity, node to node strut length, and a 106\% increase in Cn-BV/TV, with respect to the bone status at the initiation of treatment. The beneficial effects of this combined treatment derive from estrogen's ability to prevent accelerated bone resorption and, simultaneously, PTH's promotion of bone formation. These data demonstrate, in an animal model, that therapies can be devised to cure the skeletal defects associated with established osteoporosis. (J. Clin. Invest. 1993. 91:2479-2487.) Key words: bone formation - bone mineral density • calcitriol • histomorphometry • osteoporosis
\end{abstract}

\section{Introduction}

Osteoporosis is a disease characterized by low bone mass, microarchitectural deterioration of bone tissue leading to enhanced bone fragility, and a consequent increase in fracture risk (1). Two basic means of treatment have been proposed to combat bone loss in osteoporosis, namely, inhibition of bone resorption and promotion of bone formation. Estrogen (2), calcitonin (3), and bisphosphonates (4) have been successful

Address reprint requests to Dr. Victor Shen, Regional Bone Center, Helen Hayes Hospital, West Haverstraw, NY 10993.

Received for publication 19 October 1992 and in revised form 19 January 1992.

J. Clin. Invest.

(c) The American Society for Clinical Investigation, Inc.

$0021-9738 / 93 / 06 / 2479 / 09 \$ 2.00$

Volume 91, June 1993, 2479-2487 in preventing bone loss with small increments in bone mass but can not cure established osteoporosis as they do little to restore mass and trabecular connectivity. The ability to identify a regimen that can improve both bone mass and trabecular connectivity would be an important advance in treatment of the established disease. In humans, fluoride $(5,6)$, anabolic steroids (7), and intermittent administration of PTH (8-11) have been used with some success in improving cancellous bone mass. A therapeutic regimen using a combination of an antiresorptive agent, to prevent further bone loss, and an anabolic agent, to promote new bone formation, is a theoretically attractive approach for restoring bone mass. This treatment has not been rigorously tested in either humans or experimental animals.

Ovariectomy (Ovx $)^{1}$ induces osteoporosis in rats $(12,13)$ by increasing bone turnover $(14,15)$ and the rat has been successfully used as an animal model for postmenopausal osteoporosis $(16,17)$. Estrogen prevents bone loss in this animal model by reducing bone turnover (18-22) while PTH, given intermittently, acts as an anabolic agent for bone formation (23-29). In this study, we present data on changes in bone mass, as well as trabecular connectivity, as a result of treatment of Ovx animals with estrogen and/or PTH. In addition, similar treatments were initiated at three different times after Ovx in order to ascertain the effects of intervention in both preventive and curative modes.

\section{Methods}

\section{Animals and diets}

Virgin female Sprague-Dawley rats were purchased from Harlan Sprague-Dawley Inc. (Indianapolis, IN) and maintained at the Animal Research Facility of the Helen Hayes Hospital for an additional month before the start of the experiment. They were housed in a room maintained at $20^{\circ} \mathrm{C}$ on 12 -h light / $12-\mathrm{h}$ dark cycles. Bilateral ovariectomy or sham (Sham) operation were performed at the onset of the experiment when the rats were $5 \mathrm{mo}$ of age $(\approx 275 \mathrm{~g})$. The Ovx animals were fed $15 \mathrm{~g}$ per rat per $\mathrm{d}$ of Purina laboratory rodent Chow (Ralston Purina Co., St. Louis, MO), the mean food intake of the sham-operated animals, to prevent the hyperphagia associated with Ovx in rats. The experimental protocol was approved by the IACUC at Helen Hayes Hospital.

\section{Experimental protocols}

Treatment was started at three different time points: 1,3 , and 5 wk after surgery. At each time point, five different treatment groups were established: $(a)$ sham-operated animals, treated with vehicle ( $5 \%$ ethanol in corn oil and $1 \mathrm{mM} \mathrm{HCl}$ ); (b) Ovx animals, treated with vehicle; (c) Ovx animals, treated subcutaneously with $40 \mu \mathrm{g} / \mathrm{kg}$ per d of rat 1-34 PTH $(24,800 \mathrm{IU} / \mathrm{mg}$ in an in vitro rat adenoid membrane adenylate cyclase stimulation assay, Bachem Inc., Torrance, CA) dissolved in 1 $\mathrm{mM}$ of $\mathrm{HCl} ;(d) \mathrm{Ovx}$ animals, treated with $30 \mu \mathrm{g} / \mathrm{kg}$ per d of $17 \beta-$

1. Abbreviations used in this paper: Ovx, ovariectomy; Sham, shamoperated. 
estradiol dissolved in 5\% ethanol in corn oil (Sigma Chemical Co., St. Louis, MO); (e) Ovx animals, treated with a combination of PTH and estradiol. All rats were killed $4 \mathrm{wk}$ after the initiation of treatment, i.e., 5,7 , and 9 wk after surgery. Each treatment group consisted of eight animals. Two doses of demeclocycline (Lederle, Pearl River, NY) at 10 $\mathrm{mg} / \mathrm{kg}$ body weight were given to each animal 6 and $2 \mathrm{~d}$ before killing. On the day immediately before killing, the rats were housed in individual metabolic cages and a 24-h urine sample was collected. $24 \mathrm{~h}$ after the last PTH and/or estrogen injection, blood was collected via the abdominal aorta, and right tibias and femurs were excised and fixed in $70 \%$ alcohol for histomorphometric evaluation and bone mineral density measurements, respectively.

\section{Analytical procedures}

Bone mineral density. Bone mineral density of entire excised femurs was measured by dual-energy $x$-ray absorptiometry (QDR-1000, Hologic, Waltham, MA) under $5 \mathrm{~cm}$ of $70 \%$ alcohol with a 0.025 -in. collimator at the high resolution mode. Three regions of the scan from the whole femur were analyzed separately. The areas represent proximal and distal regions $25 \%$ each of total length, which contain significant amounts of cancellous bone, and the diaphysis, which represents $50 \%$ of the femoral length and is almost exclusively composed of cortical bone. Triplicate determinations of five different femurs, with a new placement after each determination, showed a coefficient of variation of the measurements at $2.5 \%$.

Blood and urine chemistry and vitamin D metabolite assays. Serum and urine calcium, phosphorus, and creatinine concentrations were measured by standard colorimetric methods as previously described. Serum $17 \beta$-estradiol was determined by radioimmunoassay using a kit from Diagnostic Products Corp. (Los Angeles, CA). The osteocalcin concentration was measured with a commercial radioimmunoassay kit (Biomedical Technologies Inc. Stoughton, MA) using rat osteocalcin as standard and a region specific domain of goat anti-rat osteocalcin antibody. Vitamin D metabolites in serum were extracted with organic solvents after chromatography on C18 Sep-Pak cartridges (Waters Associates, Milford, MA) and further purified by chromatography on silica Sep-Pak cartridges. $25(\mathrm{OH}) \mathrm{D}_{3}$ was measured by a competitive protein-binding assay using rat serum depleted of vitamin $\mathrm{D}$, as previously described $(30), 1,25(\mathrm{OH})_{2} \mathrm{D}$ was assayed by a radioreceptor assay that uses a $1,25(\mathrm{OH})_{2} \mathrm{D}$ receptor protein isolated from bovine thymus (Incstar, Stillwater, MN) (31).

Histomorphometry. Excised right tibias were dehydrated, embedded undecalcified in methyl methacrylate and sectioned longitudinally using a Jung Ultracut microtome (Reichert-Jung, Heidelberg, FRG). From the center of the tibias, $4-$ and $10-\mu \mathrm{m}$ nonconsecutive sections were obtained. The $4-\mu \mathrm{m}$ sections were stained by the Goldner's trichrome method and the $10-\mu \mathrm{m}$ sections were mounted unstained. Osteoclast surface (OC/BS), single-labeled surface (sLS/BS), and double-labeled surface ( $\mathrm{dLS} / \mathrm{BS}$ ) in the secondary spongiosa, $1 \mathrm{~mm}$ away from the growth plate, from cortex to cortex, with a total area of $\sim 2.9$ $\mathrm{mm}^{2}$, were measured directly and expressed as a percentage of the bone surface using a Merz eyepiece graticule at $\times 400$. Cancellous bone volume ( $\mathrm{Cn}-\mathrm{BV} / \mathrm{TV})$ and trabecular perimeter were measured using a direct tracing method as detailed in the following section. Trabecular thickness (Tb.Th), number (Tb.N), and separation (Tb.Sp) were calculated according to Parfitt et al. (32). Mineral apposition rate (MAR) was calculated by dividing the directly measured distance between the two tetracycline labels by the number of days between the labels. Bone formation rate $(B F R / B S)$ was calculated by $M A R *(1 / 2 s L S / B S$ $+\mathrm{dLS} / \mathrm{BS})$.

Trabecular strut analysis. The stained sections were placed under a microscope linked to an interactive measuring system (Optomax VIDS IV, Analytical Measurement Systems, Optomax Inc., Hollis, NH) by a television camera mounted on the microscope. Using an objective at a magnification of 6.3 , the section was viewed on the television screen at a total magnification of 140 . Two fields from each section, each field $1.34 \mathrm{~mm}^{2}$ in area, situated equidistant from the cortex and $1 \mathrm{~mm}$ distal to the lowest point of the growth plate, were used in the measurements.
Two sections were measured from each rat. The measurements and definitions of the parameters were a modification of those described previously by Garrahan et al. (33) and Mellish et al. (34). They included node number (N.Nd), terminus number (N.Tm), node to node strut length ( $\mathrm{Nd} . \mathrm{Nd}$ ), node to terminus strut length ( $\mathrm{Nd} . \mathrm{Tm}$ ), and terminus to terminus strut length ( $\mathrm{Tm} . \mathrm{Tm})$, all expressed per square millimeter of measured area. Each length measurement was also expressed as a percentage of the total strut length. This differed from previous measurements in that each strut that extended to the border of the field measured was given a designation of $\mathrm{Nd} . \mathrm{Nd}$, Nd.Tm, or $\mathrm{Tm}$. Tm based on the identity of structures that were immediately outside the viewing field. Total strut length (TSL) is the sum of Nd.Nd, Nd.Tm, and Tm.Tm.

Statistical analysis. Student's $t$ test was used to compare the results between Sham and Ovx + V groups. The effects of PTH and/or estrogen treatments in Ovx rats at each time point were analyzed by the method of Duncan's procedure of analysis of variance using SAS software (SAS Institute Inc., Cary, NC).

\section{Results}

\section{Serum and urine parameters}

Serum estradiol levels were $137 \pm 64 \mathrm{pmol} /$ liter ( mean \pm SD) in sham-operated groups. As expected, serum estradiol levels were $59 \pm 36 \mathrm{pmol} /$ liter in Ovx rats that were not supplemented with estradiol ( $\mathrm{Ovx}+\mathrm{V}$ and $\mathrm{Ovx}+\mathrm{P})$ while serum estradiol levels were $636 \pm 432 \mathrm{pmol} /$ liter in groups of ovariectomized rats supplemented with $17 \beta$-estradiol $(\mathrm{Ovx}+\mathrm{E}$ and $\mathrm{Ovx}+\mathrm{P}$ $+E)$. There were no significant differences in serum calcium, phosphorus, urine calcium, and phosphorus between any of the groups (data not shown). Tubular reabsorption of phosphate was the same in all but one group; Ovx $+\mathrm{E}$ treated from weeks 1 to 5 was lower (Table I).

Serum levels of $25(\mathrm{OH}) \mathrm{D}_{3}$ were not significantly different among all Ovx groups examined except that it was lower in one group $\left(\mathrm{Ovx}+\mathrm{E}\right.$, weeks 3-7). Serum levels of $1,25(\mathrm{OH})_{2} \mathrm{D}$ were decreased by $32-63 \%$ in estrogen treated groups. PTH treatment of Ovx rats resulted in a highly significant 87-113\% increase in serum $1,25(\mathrm{OH})_{2} \mathrm{D}$ concentration in each of the treatment periods. Combined PTH and estrogen treatment did not result in an increase of $1,25(\mathrm{OH})_{2} \mathrm{D}$ levels in the preventive treatment periods but did stimulate $1,25(\mathrm{OH})_{2} \mathrm{D}$ levels by 40 $120 \%$ over that of estrogen alone in the curative treatment periods. The circulating osteocalcin levels were elevated by Ovx and decreased in Ovx + E groups by $22-40 \%$. Among the Ovx groups, osteocalcin was shown to be elevated in Ovx $+\mathrm{P}$ groups by 24-58\%, and unchanged in Ovx $+\mathrm{P}+\mathrm{E}$ groups.

\section{Histomorphometric parameters and bone mineral density}

Structural parameters. As expected, cancellous bone volume in Ovx $+\mathrm{V}$ groups was $50-75 \%$ lower than respective sham groups ( Table II). The estrogen treatment alone prevented further bone loss. In these animals there was an increment of cancellous bone volume of $163 \%, 126 \%$, and $80 \%$ when compared with respective Ovx $+\mathrm{V}$ groups. PTH treatment alone also improved cancellous bone volume by $175 \%, 198 \%$, and $165 \%$ when compared with respective $\mathrm{Ovx}+\mathrm{V}$ groups at each of the three time points. Cancellous bone volume in the Ovx $+\mathrm{P}+\mathrm{E}$ group treated, in a preventive mode, from weeks 1 to 5 $(\mathrm{Ovx}+\mathrm{P}+\mathrm{E}$, weeks $1-5)$ produced a significant $240 \%$ increase of cancellous bone volume when compared with the untreated group (Ovx $+\mathrm{V}$, weeks $1-5)$ as well as a $40 \%$ increase over and above the sham-operated group (Sham, weeks 
Table I. Serum and Urine Parameters

\begin{tabular}{|c|c|c|c|c|c|}
\hline Treatment period & Treatment & $25(\mathrm{OH}) \mathrm{D}_{3}$ & $1,25(\mathrm{OH})_{2} \mathrm{D}$ & Tubular reabsorption of $\mathrm{Pi}$ & Osteocalcin \\
\hline & & nmol/liter & pmol/liter & $\%$ & $n g / m l$ \\
\hline \multirow[t]{5}{*}{ Weeks $1-5$} & $O v x+V$ & $49.9 \pm 1.5$ & $56.6 \pm 7.0^{*}$ & $93.2 \pm 1.0$ & $26.2 \pm 2.3^{8.11}$ \\
\hline & $O v x+P$ & $60.2 \pm 5.5$ & $119.3 \pm 7.4^{*}$ & $90.0 \pm 2.6$ & $32.8 \pm 2.6^{*}$ \\
\hline & $O v x+E$ & $54.4 \pm 1.7$ & $31.9 \pm 3.6^{\ddagger .8}$ & $73.9 \pm 6.2$ & $17.4 \pm 0.6^{*}$ \\
\hline & $O v x+P+E$ & $57.9 \pm 2.7$ & $30.7 \pm 3.8^{t .8}$ & $86.9 \pm 2.2$ & $22.7 \pm 1.2^{5.11}$ \\
\hline & Sham & $67.6 \pm 5.5$ & $49.7 \pm 4.3$ & $84.2 \pm 1.8$ & $23.6 \pm 0.9$ \\
\hline \multirow[t]{5}{*}{ Weeks 3-7 } & $O v x+V$ & $60.7 \pm 4.0^{\prime \prime}$ & $54.5 \pm 7.4^{\S}$ & $87.3 \pm 2.8$ & $19.7 \pm 1.2^{8.11}$ \\
\hline & $O v x+P$ & $65.9 \pm 2.7^{11}$ & $96.0 \pm 8.6^{*}$ & $88.3 \pm 1.8$ & $30.5 \pm 2.0^{*}$ \\
\hline & $O v x+E$ & $48.9 \pm 3.5^{*}$ & $36.2 \pm 2.6^{8}$ & $75.2 \pm 1.6$ & $15.1 \pm 1.5^{*}$ \\
\hline & $O v x+P+E$ & $61.7 \pm 2.5^{\prime \prime}$ & $50.6 \pm 6.2^{8}$ & $74.6 \pm 6.1$ & $21.2 \pm 1.3^{8.11}$ \\
\hline & Sham & $68.4 \pm 3.5$ & $46.3 \pm 4.3$ & $76.1 \pm 5.4$ & $20.7 \pm 1.1$ \\
\hline \multirow[t]{5}{*}{ Weeks 5-9 } & Ovx $+\mathrm{V}$ & $51.9 \pm 1.5$ & $66.2 \pm 9.1^{8,11}$ & $92.0 \pm 2.7$ & $25.2 \pm 1.4^{*}$ \\
\hline & $O v x+P$ & $58.4 \pm 4.5$ & $128.9 \pm 9.4^{*}$ & $87.6 \pm 1.8^{1}$ & $31.0 \pm 2.0^{*}$ \\
\hline & $O v x+E$ & $48.4 \pm 2.5$ & $25.0 \pm 3.1^{*}$ & $90.1 \pm 1.4$ & $15.1 \pm 0.9^{\ddagger .8}$ \\
\hline & $O v x+P+E$ & $58.4 \pm 2.7$ & $53.8 \pm 6.2^{\ddagger .11}$ & $95.6 \pm 0.8^{8}$ & $18.6 \pm 2.0^{\ddagger .8}$ \\
\hline & Sham & $64.1 \pm 4.5$ & $42.0 \pm 7.4$ & $93.2 \pm 1.3$ & $20.3 \pm 1.1$ \\
\hline
\end{tabular}

Values for Sham groups are given as a reference for comparison. $P<0.05,{ }^{*}$ vs. all other groups; ${ }^{\ddagger}$ vs. Ovx + v; ${ }^{\S} \mathrm{vs.} \mathrm{Ovx} \mathrm{+} \mathrm{P;} "$ vs. Ovx + E; 'vs. Ovx + P + E.

1-5) (Table II). The group treated with the combination of PTH and estrogen, when administered in a curative mode from weeks 5 to 9 (Ovx $+P+E$, weeks 5-9), showed a 225\% increase of cancellous bone volume when compared with the untreated Ovx $+\mathrm{V}$ group killed on the same date $(\mathrm{Ovx}+\mathrm{V}$, weeks 5-9) (Table II) and a 106\% increase when compared with the untreated $O v x+V$ group killed at the time of the initiation of treatment for this group (Ovx $+\mathrm{V}$, weeks 1-5) (Table II and Fig. 1 a). Ovx + P + E through weeks 5-9 restored $84 \%$ of the cancellous bone volume relative to Sham, weeks $5-9$, in contrast to a $74 \%$ loss of cancellous bone volume in the Ovx + V, weeks 5-9 rats. Combined PTH and estrogen treatment resulted in improved cancellous bone volume when compared with either PTH or estrogen treatment alone. However, the differences between Ovx $+\mathrm{P}+\mathrm{E}$ and $\mathrm{Ovx}+\mathrm{P}$ at weeks 3-7 and 5-9 were not statistically significant. Mean trabecular thickness was similar in Sham, Ovx $+\mathrm{V}$, and Ovx $+\mathrm{E}$ groups. All PTH treatments, whether Ovx $+\mathrm{P}$ or Ovx $+\mathrm{P}+\mathrm{E}$, increased the trabecular thickness by $25-40 \%$ (Table II). The average number of trabecular plates per square millimeter showed a time-dependent decrease in Ovx $+\mathrm{V}$ groups from 4.2 before surgery to $2.0,1.9$, and 1.2 at 5,7 , and 9 wk after surgery,

Table II. Effects of Treatments on Structural Parameters of Cancellous Bone

\begin{tabular}{|c|c|c|c|c|c|}
\hline Treatment period & Treatment & $\mathrm{Cn}-\mathrm{BV} / \mathrm{TV}$ & Tb.Th & Tb.N & Tb.Sp \\
\hline & & $\%$ & $\mu m$ & & $\mu m$ \\
\hline \multirow[t]{5}{*}{ Weeks $1-5$} & $O v x+v$ & $8.2 \pm 1.1^{*}$ & $40.3 \pm 2.5^{*}$ & $2.0 \pm 0.1^{*}$ & $483 \pm 11^{*}$ \\
\hline & $\mathrm{Ovx}+\mathrm{P}$ & $22.6 \pm 2.7^{\ddagger .1}$ & $57.8 \pm 3.1^{\ddagger .11}$ & $3.8 \pm 0.2^{\ddagger}$ & $210 \pm 24^{\ddagger}$ \\
\hline & $O v x+E$ & $21.6 \pm 1.4^{\ddagger .1}$ & $49.7 \pm 3.3^{*}$ & $4.3 \pm 0.1^{\ddagger}$ & $181 \pm 8^{\ddagger}$ \\
\hline & $O v x+P+E$ & $27.9 \pm 1.1^{*}$ & $65.7 \pm 2.8^{\ddagger .11}$ & $4.3 \pm 0.1^{\ddagger}$ & $171 \pm 12^{\ddagger}$ \\
\hline & Sham & $19.8 \pm 1.1$ & $43.4 \pm 1.7$ & $4.5 \pm 0.2$ & $178 \pm 11$ \\
\hline \multirow[t]{5}{*}{ Weeks 3-7 } & $O v x+V$ & $6.9 \pm 0.8^{*}$ & $35.7 \pm 2.4^{*}$ & $1.9 \pm 0.1^{*}$ & $519 \pm 60^{*}$ \\
\hline & $O v x+P$ & $20.6 \pm 0.7^{ \pm .11}$ & $56.5 \pm 1.5^{\ddagger .11}$ & $3.6 \pm 0.1^{\ddagger}$ & $219 \pm 9^{\ddagger}$ \\
\hline & $O v x+E$ & $15.6 \pm 1.5^{*}$ & $46.5 \pm 1.3^{*}$ & $3.3 \pm 0.3^{\ddagger}$ & $273 \pm 34^{\ddagger}$ \\
\hline & $O v x+P+E$ & $20.6 \pm 1.4^{\ddagger .11}$ & $56.9 \pm 2.1^{\ddagger . \|}$ & $3.6 \pm 0.2^{\ddagger}$ & $228 \pm 21^{\ddagger}$ \\
\hline & Sham & $20.3 \pm 0.9$ & $46.4 \pm 1.8$ & $4.3 \pm 0.1$ & $182 \pm 7$ \\
\hline \multirow[t]{5}{*}{ Weeks 5-9 } & $O v x+v$ & $5.2 \pm 2.0^{8.1}$ & $40.8 \pm 3.5^{5.1}$ & $1.2 \pm 0.3^{*}$ & $1343 \pm 462^{*}$ \\
\hline & $O v x+P$ & $13.8 \pm 1.9^{\ddagger}$ & $51.3 \pm 4.5^{\ddagger .11}$ & $2.6 \pm 0.2^{\ddagger}$ & $354 \pm 43^{\ddagger}$ \\
\hline & Ovx $+E$ & $9.4 \pm 1.1^{\top}$ & $38.5 \pm 1.8^{5.1}$ & $2.4 \pm 0.2^{\ddagger .1}$ & $398 \pm 47^{\ddagger}$ \\
\hline & $O v x+P+E$ & $16.9 \pm 1.5^{\ddagger .11}$ & $51.4 \pm 1.5^{\ddagger .11}$ & $3.2 \pm 0.2^{\ddagger .11}$ & $269 \pm 25^{\ddagger}$ \\
\hline & Sham & $20.2 \pm 1.4$ & $47.0 \pm 2.3$ & $4.2 \pm 0.2$ & $189 \pm 11$ \\
\hline
\end{tabular}

Values for Sham groups are given as a reference for comparison. $P<0.05,{ }^{*}$ vs. all other groups; ${ }^{\ddagger}$ vs. Ovx + V; vs. Ovx + P; " vs. Ovx + E; 'vs. Ovx + P + E. 

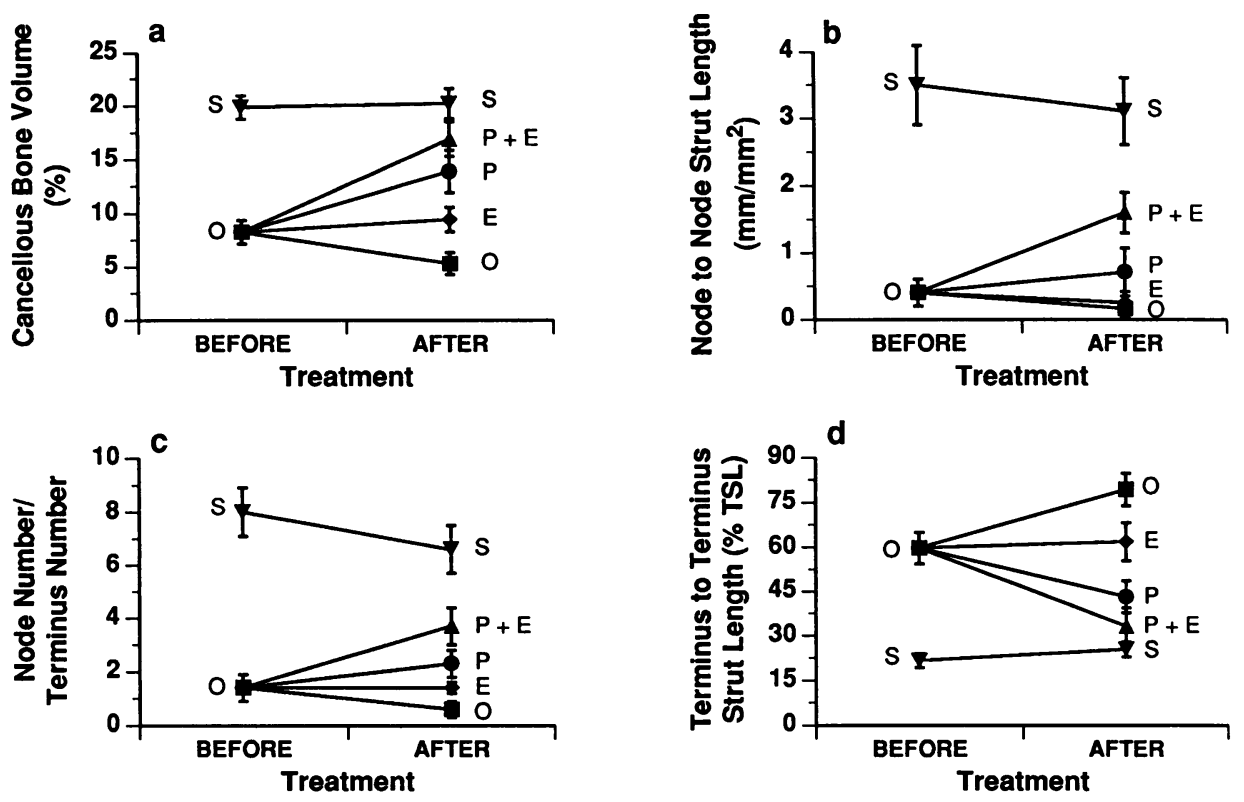

Figure 1. Static and dynamic indices, before and after PTH and/or estrogen treatments, in Ovx rats with established osteopenia. 5-moold virgin female rats were either Sham-operated or ovariectomized. Two groups of rats, Sham and Ovx,
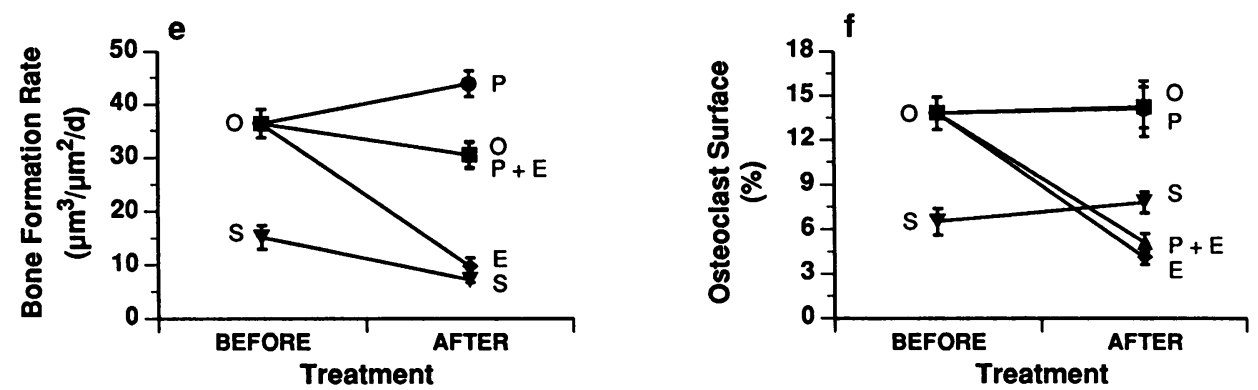
were killed before the treatment and at week 5 after the operations. Four groups of Ovx rats were further treated with vehicle, PTH, estrogen or PTH plus estrogen from weeks 5 to 9 . They were killed after the treatment at week 9. One Sham group was also killed at the same time for comparison purpose. The histomorphometric analysis, strut analysis, and bone mineral density measurement of the killed rats were as described in the materials and methods. ( $a$ ) Cancellous bone volume; $(b)$ node to node strut length; (c) node number/terminus number; $(d)$ percentage of terminus to terminus strut length to total strut length; $(e)$ bone formation rate; $(f)$ osteoclast surface; $(g)$ bone mineral density of the distal femur; $(h)$ bone mineral density of the diaphyseal

femur.

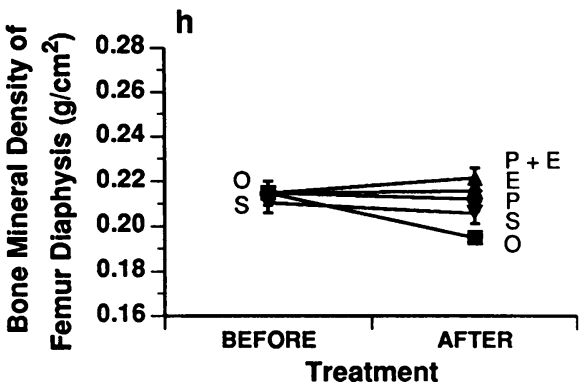

respectively. Each of the treatment groups showed significant preservation of plate number ( Table II ). For example, Ovx $+\mathbf{P}$ $+E$, weeks 5-9 showed a $166 \%$ increase when compared with Ovx $+V$, weeks 5-9 and a $60 \%$ increase when compared with the Ovx $+\mathrm{V}$ group killed at the time of the initiation of treatment for this treatment group (Ovx $+\mathrm{V}$, weeks 1-5). Conversely, average plate separation increased rapidly with time in all Ovx $+V$ from $189 \mu \mathrm{m}$ before surgery to 483,519 , and 1,343 $\mu \mathrm{m}$ at 5,7 , and $9 \mathrm{wk}$ after surgery, respectively. Each treatment group displayed a lower degree of plate separation than the Ovx + V groups; the combined treatment with PTH and estrogen group showed the lowest degree of separation at all time points (Table II).

Trabecular strut analysis. Three parameters which reflect trabecular connectivity, node to node strut length, node number, and node to terminus ratio, showed a significant decrease after ovariectomy ( Table III ). In Ovx + V rats, only $11 \%, 4 \%$, and $6 \%$ of node to node strut length were retained when compared to Sham at weeks 5, 7, and 9, respectively. PTH or estrogen alone helped to retain more node to node strut length (Ovx + P: $71 \%, 77 \%$, and $23 \%$; Ovx + E: $91 \%, 38 \%$, and $10 \%$ at each of the time points, respectively). Rats treated with combined PTH and estrogen (Ovx $+\mathrm{P}+\mathrm{E}$, weeks 1-5), given in a preventive mode, retained $137 \%$ of their node to node strut length, a 1,100\% improvement when compared with Ovx $+\mathrm{V}$, weeks 1-5. When rats were treated with PTH and estrogen in a curative mode (Ovx $+P+E$, weeks 5-9), they retained $52 \%$ of their node to node strut length. This is an improvement of $700 \%$ when compared with Ovx + V killed after the same duration after Ovx (Ovx + V, weeks 5-9) and a 300\% increase when compared with Ovx $+\mathrm{V}$ killed at the time of initiation of treatment (Ovx $+\mathrm{V}$, weeks 1-5) (Table III and Fig. $1 b)$. The other connectivity parameters, node number and ratio of node number to terminus number, showed a similar pattern (Table III 
Table III. Effects of Treatments on Trabecular Connectivity

\begin{tabular}{|c|c|c|c|c|c|}
\hline \multirow[b]{2}{*}{ Treatment period } & \multirow[b]{2}{*}{ Treatment } & \multicolumn{3}{|c|}{ Connectivity indices } & \multirow{2}{*}{$\begin{array}{l}\text { Disconnectivity indices } \\
\mathrm{Tm} . \mathrm{Tm} / \mathrm{TSL}\end{array}$} \\
\hline & & Nd to Nd length & Node number & $\mathrm{Nd}$ to $\mathrm{Tm}$ ratio & \\
\hline & & $\mathrm{mm} / \mathrm{mm}^{2}$ & No. $/ \mathrm{mm}^{2}$ & & $\%$ \\
\hline \multirow[t]{5}{*}{ Weeks $1-5$} & $O v x+V$ & $0.4 \pm 0.2^{*}$ & $1.4 \pm 0.5^{*}$ & $0.10 \pm .04^{*}$ & $59 \pm 7^{*}$ \\
\hline & $O v x+P$ & $2.5 \pm 0.6^{\ddagger, 1}$ & $5.8 \pm 0.7^{\ddagger .1}$ & $0.36 \pm .05^{\ddagger .1}$ & $30 \pm 5^{\ddagger}$ \\
\hline & $O v x+E$ & $3.2 \pm 0.7^{\ddagger .1}$ & $7.1 \pm 0.8^{\ddagger}$ & $0.41 \pm .06^{\ddagger, 1}$ & $24 \pm 7^{\ddagger}$ \\
\hline & $O v x+P+E$ & $4.8 \pm 0.6^{*}$ & $7.7 \pm 0.6^{\ddagger .1}$ & $0.65 \pm .06^{*}$ & $8 \pm 1^{*}$ \\
\hline & Sham & $3.5 \pm 0.6$ & $8.9 \pm 1.3$ & $0.42 \pm .06$ & $21 \pm 2$ \\
\hline \multirow[t]{5}{*}{ Weeks 3-7 } & $O v x+V$ & $0.1 \pm 0.1^{*}$ & $0.9 \pm 0.2^{*}$ & $0.06 \pm .01^{*}$ & $69 \pm 4^{*}$ \\
\hline & $O v x+P$ & $2.0 \pm 0.2^{\ddagger .11}$ & $3.7 \pm 0.4^{\ddagger}$ & $0.26 \pm .03^{\ddagger}$ & $33 \pm 2^{\ddagger}$ \\
\hline & $O v x+E$ & $1.0 \pm 0.3^{ \pm .8}$ & $3.3 \pm 0.5^{\ddagger}$ & $0.21 \pm .03^{\ddagger .1}$ & $39 \pm 5^{\ddagger}$ \\
\hline & $O v x+P+E$ & $1.8 \pm 0.4^{\ddagger}$ & $4.2 \pm 0.8^{\ddagger}$ & $0.32 \pm .05^{\ddagger .11}$ & $29 \pm 3^{\ddagger}$ \\
\hline & Sham & $2.6 \pm 0.5$ & $6.0 \pm 0.9$ & $0.30 \pm .05$ & $31 \pm 4$ \\
\hline \multirow[t]{5}{*}{ Weeks 5-9 } & $O v x+V$ & $0.2 \pm 0.1^{1}$ & $0.6 \pm 0.3^{5,1}$ & $0.06 \pm .03^{5,1}$ & $79 \pm 9^{5,1}$ \\
\hline & $O v x+P$ & $0.7 \pm 0.4^{\prime}$ & $2.3 \pm 0.5^{\ddagger}$ & $0.21 \pm .05^{\ddagger . \|}$ & $43 \pm 5^{\ddagger}$ \\
\hline & $O v x+E$ & $0.3 \pm 0.2^{1}$ & $1.4 \pm 0.2^{\prime}$ & $0.09 \pm .02^{\delta, 1}$ & $61 \pm 6^{\prime}$ \\
\hline & $O v x+P+E$ & $1.6 \pm 0.3^{*}$ & $3.7 \pm 0.7^{\ddagger .11}$ & $0.27 \pm .05^{\ddagger . .1}$ & $33 \pm 1^{\ddagger .11}$ \\
\hline & Sham & $3.1 \pm 0.5$ & $6.6 \pm 0.9$ & $0.41 \pm .05$ & $19 \pm 5$ \\
\hline
\end{tabular}

Values for Sham groups are given as a reference for comparison. $P<0.05,{ }^{*}$ vs. all other groups; ${ }^{\ddagger}$ vs. Ovx + V; ${ }^{\S}$ vs. Ovx + P; " vs. Ovx + E; vs. Ovx + P + E.

and Fig. $1 \mathrm{c}$ ). The parameter most reflecting loss of connectivity, percentage terminus to terminus strut length to total strut length, showed an increase of $81 \%, 123 \%$, and $140 \%$ after ovariectomy when compared with the Sham groups at each time point, respectively (Table III and Fig. $1 d$ ). Each of the treatment groups displayed a greater degree of connectivity in trabecular structure. The combined PTH and estrogen treatment was the most effective in improving connectivity.

Cellular activity parameters. Osteoclast surface, as a percentage of bone surface, was elevated after Ovx (112\%, 87\%, and $82 \%$ higher than Sham at weeks 5,7 , and 9, respectively) and estrogen reversed this increase $(-71 \%,-56 \%$, and $-71 \%$ of Ovx $+\mathrm{V}$ at weeks 5, 7, and 9, respectively). PTH treatment alone did not further elevate osteoclast surface in Ovx rats nor did it override the suppressive effects of estrogen $(-64 \%,-72 \%$ and $-64 \%$ at weeks 5,7 , and 9 , respectively) (Table IV). The combined PTH and estrogen was similar to estrogen treatment alone.

The bone formation rate was elevated after ovariectomy $(139 \%, 186 \%$, and $324 \%$ at weeks 5,7 , and 9 , respectively)

Table IV. Effects of Treatments on Cellular Activities

\begin{tabular}{|c|c|c|c|c|c|}
\hline Treatment period & Treatment & $\begin{array}{l}\text { Osteoclast surface } \\
\text { (OC/BS\%) }\end{array}$ & $\begin{array}{l}\text { Total labeling surface } \\
\text { (TLS/BS) }\end{array}$ & Mineral apposition rate & Bone formation rate \\
\hline & & & & $\mu m / d$ & $\mu m^{3} / \mu m^{2}$ per $d$ \\
\hline \multirow[t]{5}{*}{ Weeks 1-5 } & $O v x+v$ & $13.8 \pm 1.1^{*}$ & $25.5 \pm 1.5^{\prime \prime}$ & $1.477 \pm 0.106^{\| \prime}$ & $36.4 \pm 3.7^{8,11}$ \\
\hline & $O v x+P$ & $9.2 \pm 0.7^{*}$ & $34.5 \pm 5.4^{\prime \prime}$ & $1.513 \pm 0.054^{\prime \prime}$ & $50.8 \pm 7.1^{\ddagger, \|}$ \\
\hline & $O v x+E$ & $4.0 \pm 0.4^{\ddagger . \S}$ & $8.5 \pm 1.1^{11}$ & $1.156 \pm 0.059^{\ddagger .1}$ & $9.7 \pm 1.1^{*}$ \\
\hline & $\mathrm{Ovx}+\mathrm{P}+\mathrm{E}$ & $5.0 \pm 0.5^{\ddagger, \S}$ & $34.1 \pm 2.5^{*}$ & $1.386 \pm 0.061^{\| \prime}$ & $46.8 \pm 2.8^{\prime \prime}$ \\
\hline & Sham & $6.5 \pm 0.9$ & $12.4 \pm 1.2$ & $1.191 \pm 0.066$ & $15.2 \pm 2.2$ \\
\hline \multirow[t]{5}{*}{ Weeks 3-7 } & $O v x+v$ & $12.7 \pm 1.5^{11,1}$ & $24.7 \pm 1.8^{11}$ & $1.055 \pm 0.086^{\S, 1}$ & $26.0 \pm 3.5^{*}$ \\
\hline & $O v x+P$ & $11.0 \pm 2.5^{11,1}$ & $27.2 \pm 3.2^{11}$ & $1.540 \pm 0.152^{\ddagger}$ & $42.8 \pm 8.3^{\ddagger . \|}$ \\
\hline & $O v x+E$ & $5.6 \pm 1.0^{\ddagger .8}$ & $8.3 \pm 1.2^{*}$ & $1.288 \pm 0.075$ & $11.6 \pm 0.9^{*}$ \\
\hline & $\mathrm{Ovx}+\mathbf{P}+\mathrm{E}$ & $3.5 \pm 0.7^{\ddagger .8}$ & $27.1 \pm 1.6^{11}$ & $1.478 \pm 0.095^{\ddagger}$ & $39.7 \pm 2.9^{\ddagger .11}$ \\
\hline & Sham & $6.8 \pm 1.9$ & $7.2 \pm 1.6$ & $1.318 \pm 0.096$ & $9.1 \pm 2.5$ \\
\hline \multirow[t]{5}{*}{ Weeks 5-9 } & $O v x+v$ & $14.2 \pm 1.4^{\| 1,1}$ & $26.9 \pm 1.6^{11}$ & $1.172 \pm 0.115$ & $30.5 \pm 2.3^{\prime \prime}$ \\
\hline & Ovx $+P$ & $14.1 \pm 1.9^{\|, 1}$ & $28.3 \pm 6.6^{11}$ & $1.325 \pm 0.100$ & $43.9 \pm 9.4^{\prime \prime}$ \\
\hline & $O v x+E$ & $4.1 \pm 0.5^{\ddagger . \S}$ & $9.0 \pm 1.4^{*}$ & $1.107 \pm 0.069$ & $9.7 \pm 1.6^{*}$ \\
\hline & $\mathrm{Ovx}+\mathrm{P}+\mathrm{E}$ & $5.1 \pm 0.6^{\ddagger . \S}$ & $23.3 \pm 2.7^{\prime \prime}$ & $1.315 \pm 0.133$ & $30.5 \pm 2.5^{\| \prime}$ \\
\hline & Sham & $7.8 \pm 0.7$ & $6.8 \pm 0.7$ & $1.075 \pm 0.087$ & $7.2 \pm 0.5$ \\
\hline
\end{tabular}

Values for Sham groups are given as a reference for comparison. $P<0.05,{ }^{*}$ vs. all other groups; ${ }^{\ddagger}$ vs. Ovx + v; ${ }^{\S}$ vs. Ovx $+\mathrm{P} ; \quad \| v s$. Ovx $+\mathrm{E}$; "vs. Ovx + P + E. 
when compared to Shams and severely inhibited by estrogen replacement when compared with Ovx $+\mathrm{V}(-72 \%,-55 \%$, and $-68 \%$ at weeks 5,7 , and 9 , respectively) (Table IV). PTH increased the bone formation rate in Ovx animals, when give alone, by $40 \%, 65 \%$, and $44 \%$ when compared with Ovx $+\mathrm{V}$ groups. Administration of PTH with estrogen dramatically increased bone formation rate by $382 \%, 242 \%$, and $214 \%$ when compared with estrogen alone. The changes in bone formation rate were mainly affected by the changes in total labeled surface. There was an indication of a small increase in bone mineral apposition rate by PTH treatment but the trend was not consistent. There were no noticeable differences in the pattern of changes in bone formation rate or osteoclast surface whether the treatment was given in a preventive or curative mode. The cellular activity before and after treatments from 5 to $9 \mathrm{wk}$ are shown in Fig. $1, e$ and $f$.

Bone mineral density. Bone mineral density measurements of the distal region of the femurs, enriched with cancellous bone, paralleled the cancellous bone volume results obtained from the proximal tibia (Table $\mathrm{V}$ and Fig. $1 \mathrm{~g}$ ). As expected, bone mineral density of the distal femurs in Ovx $+\mathrm{V}$ groups was $11-17 \%$ lower than respective Sham groups (Table V). PTH treatment alone improved bone mineral density by $9 \%$, $13 \%$, and $15 \%$ when compared with respective Ovx $+\mathrm{V}$ groups at each of the three time points, respectively. The estrogen treatment alone prevented further bone loss and resulted in an increment of bone mineral density at the distal femur of $12 \%$, $15 \%$, and $16 \%$ when compared with respective Ovx $+V$ groups. Bone mineral density of the distal femur in the Ovx $+\mathrm{P}$ + E group, treated in a preventive mode, from weeks 1 to 5 (Ovx $+\mathrm{P}+\mathrm{E}$, weeks 1-5), exhibited a $36 \%$ increase in bone mineral density when compared with the untreated group $(\mathrm{Ovx}+\mathrm{V}$, weeks 1-5). The Ovx $+\mathrm{P}+\mathrm{E}$ groups treated in a

Table V. Effects of Treatments on Bone Mineral Density of the Femurs

\begin{tabular}{clll}
\hline & & \multicolumn{2}{c}{ Bone mineral density } \\
\cline { 3 - 4 } $\begin{array}{c}\text { Treatment } \\
\text { period }\end{array}$ & \multicolumn{1}{c}{ Treatment } & \multicolumn{1}{c}{ Distal region } & Diaphyseal region \\
\hline & & \multicolumn{2}{c}{$g / \mathrm{cm}^{2}$} \\
Weeks 1-5 & Ovx + V & $0.2142 \pm 0.0051^{*}$ & $0.2148 \pm 0.0054$ \\
& Ovx + P & $0.2339 \pm 0.0032^{\ddagger, 1}$ & $0.2110 \pm 0.0044$ \\
& Ovx + E & $0.2400 \pm 0.0051^{\ddagger, 1}$ & $0.2115 \pm 0.0036$ \\
& Ovx + P + E & $0.2579 \pm 0.0054^{*}$ & $0.2018 \pm 0.0055$ \\
Weeks 3-7 & Sham & $0.2404 \pm 0.0047$ & $0.2105 \pm 0.0045$ \\
& Ovx + V & $0.2007 \pm 0.0048^{*}$ & $0.2007 \pm 0.0027$ \\
& Ovx + P & $0.2275 \pm 0.0023^{\ddagger, 1}$ & $0.2056 \pm 0.0023$ \\
& Ovx + E & $0.2317 \pm 0.0046^{\ddagger, 1}$ & $0.2041 \pm 0.0031$ \\
& Ovx + P + E & $0.2543 \pm 0.0038^{*}$ & $0.2059 \pm 0.0029$ \\
& Sham & $0.2348 \pm 0.0041$ & $0.2084 \pm 0.0062$ \\
Weeks 5-9 & Ovx + V & $0.1940 \pm 0.0042^{*}$ & $0.1950 \pm 0.0024^{*}$ \\
& Ovx + P & $0.2237 \pm 0.0056^{\ddagger, 1}$ & $0.2122 \pm 0.0053^{\ddagger}$ \\
& Ovx + E & $0.2250 \pm 0.0040^{\ddagger, 1}$ & $0.2158 \pm 0.0045^{\ddagger}$ \\
& Ovx + P + E & $0.2566 \pm 0.0038^{*}$ & $0.2215 \pm 0.0047^{\ddagger}$ \\
& Sham & $0.2346 \pm 0.0034$ & $0.2059 \pm 0.0046$
\end{tabular}

Values for Sham groups are given as a reference for comparison. $P$ $<0.05,{ }^{*}$ vs. all other groups; ${ }^{\ddagger}$ vs. Ovx $+\mathrm{V} ;{ }^{\S}$ vs. Ovx $+\mathrm{P} ; "$ "vs. $\mathrm{Ovx}+\mathrm{E} ;{ }^{\prime}$ vs. Ovx + P + E. curative mode, from weeks 5 to 9 (Ovx $+P+E$, weeks 5-9), showed a $32 \%$ increase of bone mineral density at the distal femur when compared with untreated Ovx $+\mathrm{V}$ groups sacrificed on the same date (Ovx $+V$, weeks 5-9) and a $29 \%$ increase when compared with untreated Ovx $+\mathrm{V}$ group sacrificed at the time of initiation of treatment for this group (Ovx $+\mathrm{V}$, weeks 1-5) (Table V and Fig. 1 g). Combined PTH and estrogen treatment resulted in a significantly improved bone mineral density of the distal femur when compared with either PTH or estrogen treatment alone (Table V). The diaphyseal region of the femurs, enriched with cortical bone, showed little change in bone mineral density regardless of the time and type of treatment ( Table V).

\section{Discussion}

The importance of developing a therapeutic regimen that restores lost bone mass and trabecular structure in postmenopausal osteoporosis cannot be overly stressed. Whereas human studies to this end are tedious and time consuming, one can more rapidly and safely explore new therapeutic regimens by performing controlled animal studies. While there are some differences in the effects of estrogen deficiency on the skeleton of humans and rats, there are sufficient similarities between the responses of the two species to warrant use of this model to provide preliminary evaluation of a potential therapeutic approach $(16,17)$. In this study, we have evaluated the effects of administering $17 \beta$-estradiol, intermittent PTH, or a combination of both agents on bone mass and structure in ovariectomized rats. When PTH and estrogen were given in a preventive (from week 1) or curative (from week 3 or 5 post-Ovx) mode, both prevented estrogen deficiency-induced bone loss. The combination of PTH and estrogen treatment was superior to the use of either agent alone to increase mass and improve bone structure. The most dramatic impact of combined PTH and estrogen treatment was seen when they were given in a curative mode to rats with established osteoporosis. Our results demonstrate the possibility of a significant restoration of both cancellous bone volume and trabecular connectivity with combined use of PTH and estrogen.

Blood and urine chemistry were found to be nondiscriminating, as is the case in human studies. Although the differences among groups did not reach statistical difference, the tubular reabsorption of phosphate, in general, is higher in Ovx rats and lower in estrogen-treated Ovx rats, a situation similar to that reported in humans (35). The reason for the lack of phosphaturic effect of the PTH injection is probably due to the long interval between PTH administration and urine collection. Increased $1,25(\mathrm{OH})_{2} \mathrm{D}$ levels after estrogen treatment in postmenopausal women has been proposed to be a possible mechanism of estrogen action in the prevention of bone loss $(35,36)$. In ovariectomized rats, estrogen treatment resulted in either no change or inhibitory effects on the serum levels of $1,25(\mathrm{OH})_{2} \mathrm{D}$ while loss of bone mass was prevented (37). Thus, our results and others (37) indicate that, whereas the rat model mimics some of the sex steroid effects on bone, it is not ideal for studying the possible role of $1,25(\mathrm{OH})_{2} \mathrm{D}$ in mediating the positive effects of estrogen on the skeleton. As expected, PTH treatment alone increased $1,25(\mathrm{OH})_{2} \mathrm{D}$ above that of Sham or Ovx $+\mathrm{V}$ groups. 1,25( $\mathrm{OH})_{2} \mathrm{D}$ levels were higher in the combined treatment groups than in the estrogen treatment 
groups, presumably the result of the administration of PTH. Increased levels of $1,25(\mathrm{OH})_{2} \mathrm{D}$ have also been proposed to be related to the anabolic action of PTH (38) but a direct causeeffect relationship cannot be determined at this time. The pattern of osteocalcin expression appears to be related to the bone formation rate in our study. This is not surprising as it has been shown that the level of osteocalcin is positively correlated with the bone formation rate in humans $(39,40)$. Increased osteocalcin has been linked to increased turnover in rats after Ovx as well $(41,42)$. Whether the increased osteocalcin in PTHtreated groups is the result of increased $1,25(\mathrm{OH})_{2} \mathrm{D}$ cannot be determined but $1,25(\mathrm{OH})_{2} \mathrm{D}$ has been shown to stimulate osteocalcin expression in vitro (43).

Bone loss resulting from estrogen deficiency has been studied by bone mineral density and histomorphometric methods in rats and humans. We and others have previously shown by conventional methods of bone mass measurement, in humans and rats, that estrogen can prevent such bone loss. We have confirmed these observations by showing that estrogen can prevent further bone loss whether given early, in a preventive mode, or late, in a curative mode.

Whereas the total cancellous bone mass is important, the infrastructure of the cancellous bone lattice, or its degree of connectivity, may be equally or more important in determining its mechanical competence. Several techniques are now in use to analyze trabecular microarchitecture. Direct $(44,45)$ and indirect $(32,46)$ measurement of trabecular plate thickness, separation, and number have been used extensively. Less common methods to measure marrow and trabecular star volume (47) and trabecular bone pattern factors (48) are also available. Recently, a method for the analysis of two-dimensional trabecular structure was described by Garrahan et al. (33), and adapted to a manual method in our laboratory (34). Amongst the parameters measured, node number, node to node strut length, and node to terminus ratio are measures of connectivity of the trabecular plates while terminus to terminus strut length, as a percentage of total strut length, is an indicator of loss of connectivity (49). We had demonstrated that ovariectomy $(\mathrm{Ovx}+\mathrm{V})$ reduces all indices of connectivity and increases the indices of disconnectivity and that estrogen replacement in these animals is capable of preventing the loss of connectivity. Based on the assumption that loss of connectivity precedes the loss of bone mass, such structural measurements would be a more sensitive indicator of bone destruction than bone mass measurements. This concept is supported by our findings that changes in connectivity parameters are more rapid and, thus, more severe than changes in bone mass parameters in Ovx animals.

Although the continuous elevation of PTH has been considered catabolic in nature, the intermittent administration of PTH has been shown, by conventional bone mass measurements, to illicit an anabolic response in controlled rat $(24,26$, $28,29,38,50-52)$ and uncontrolled human (53-55) studies. We have similarly found that administration of PTH increased the bone formation rate and moderately increased trabecular thickness without affecting the osteoclast surface. The net result of these actions is the preservation of bone mass. The combination of PTH and estrogen showed the most dramatic improvement in cancellous bone volume. This was achieved by the simultaneous maintenance of a high bone formation rate, through the anabolic action of $\mathrm{PTH}$, and a marked inhibition of bone resorption by estrogen. The success of this combined treatment in improving bone mass, whether the treatment was initiated immediately after ovariectomy, as a preventive measure, or after significant bone loss had occurred, as a curative measure, has demonstrated the possible clinical utility of this treatment regimen. This regimen may be extremely useful in the clinical setting as patients often present themselves only after significant bone loss has occurred.

While PTH $($ Ovx + P) and estrogen $($ Ovx + E) treatment alone preserved trabecular connectivity, the parameters of connectivity were significantly improved by combined PTH and estrogen administration ( $\mathrm{Ovx}+\mathrm{P}+\mathrm{E})$, when compared with the vehicle-treated group killed at the same time or the vehicletreated group killed before treatment. This result implies that the additional bone mass accrued by PTH and estrogen treatment is not simply due to trabecular thickening or to de novo synthesis of isolated trabeculae, a situation where one might find increased trabecular number or thickness without increased node number and node to node strut length. The mechanism of trabecular reconnection is currently under investigation. Whether the new bone growth resulted in the same trabecular configuration and equivalent mechanical competence as that presented before the bone loss still requires to be determined. The mechanical competence of rat vertebral bodies treated with PTH alone has been reported to increase significantly when compared to vertebrae in untreated rats (56) and, thus, it is likely that the mechanical integrity of the new bone will be retained in rats treated with PTH plus estrogen. The improvement in cancellous bone volume is corroborated by an improvement of the bone mineral density in the cancellous bone-enriched distal region, but not the cortical-bone enriched diaphyseal region, of the femurs. Although our results were derived from long bones, there have been reports of comparable observations in cancellous bone of the axial skeleton, such as the vertebra, both in estrogen- or PTH-treated animals ( 51 , $56,57)$. Therefore, it is likely that our findings may be valid for other trabecular sites as well, although this awaits confirmation.

One concern regarding the use of PTH as an anabolic agent for cancellous bone is that the increase in cancellous bone volume could be achieved at the expense of cortical bone, as suggested in a previous human study (10). Slovik et al. (54) theorized that if a person does not have a robust $1,25-(\mathrm{OH})_{2}-\mathrm{D}$ response to $\mathrm{PTH}$, intestinal calcium absorption will not increase appropriately, and the calcium demands for trabecular bone formation will be met at the expense of cortical bone. Thus, in ensuing studies, $1,25-(\mathrm{OH})_{2} \mathrm{D}$ was given together with PTH to prevent the possible loss of cortical bone. As an alternative to the above approach, we decided to combine an antiresorber, estrogen, with PTH in order to protect against cortical bone loss. This takes advantage of the idea that estrogen has been shown to be very effective in preventing cortical bone loss (2) and also increases $1,25-(\mathrm{OH})_{2}$-D levels in humans (35). In short-term rat studies by Gunness-Hey and Hock (24), PTH has been shown to increase cancellous bone without reducing cortical bone. We have confirmed their findings in this study. The rat model may differ from humans because of the high calcium content in the diet, an exuberant $1,25-(\mathrm{OH})_{2} \mathrm{D}$ response to PTH and the better efficiency of intestinal calcium absorption due to the relatively young age of the animals. A similar study with aged animals, given a moderate calcium diet, may be more appropriate in deciphering the role of 1,25 $(\mathrm{OH})_{2} \mathrm{D}$ and / or estrogen in preventing cortical bone loss. 
Although our findings are exciting, these experiments only address some of the important issues and many questions remain to be answered. For example, what are the prolonged effects of such combined treatment, does bone restored by combined treatment remain structurally intact after the treatment is terminated, or will continued estrogen supplementation alone will be sufficient to maintain the restored bone? Nevertheless, our results strongly support the possibility of restoring bone health in established postmenopausal osteoporotic subjects with a combined PTH and estrogen treatment. A controlled clinical trial using the combination of estrogen and PTH is clearly well warranted.

\section{Acknowledgments}

The authors acknowledge the outstanding technical assistance of Ms. W. Horbert, M. Schnitzer, and C. Mars.

This study was supported by National Institutes of Health grant AR39191.

\section{References}

1. Bouillon, R., P. Burkhardt, C. Christiansen, H. A. Fleisch, T. Fujita, C. Gennari, T. J. Martin, G. Mazzuoli, L. J. Melton, J. D. Ringe, et al. 1991. Concensus Development Conference: Prophylaxis and treatment of osteoporosis. Osteoporosis Int. 1:114-117.

2. Lindsay, R., D. M. Hart, J. M. Aitken, E. B. MacDonald, J. B. Anderson, and A. C. Clarke. 1976. Long term prevention of postmenopausal osteoporosis by estrogen. Lancet. 1:1038-1040.

3. Consoli, V., P. Alfieri, C. Giuntini, M. Manca, F. Avaldi, and R. Soncini. 1991. A double-blind placebo-controlled trial of the efficacy and tolerability of intranasal elcatonin administered to patients suffering from senile and postmenopausal osteoporosis. Curr. Ther. Res. 50:369-377.

4. Storm, T., G. Thamsborg, T. Steiniche, H. K. Genant, and O. H. Soresen. 1990. Effect of intermittent cyclical etidronate therapy on bone mass and fracture rate in women with postmenopausal osteoporosis. N. Engl. J. Med. 322:12651271.

5. Riggs, B. L., E. Seemon, S. F. Hodgeson, D. R. Taves, and W. M. O'Fallon. 1982. Effect of the fluoride/calcium regimen on vertebral fracture occurrence in postmenopausal osteoporosis. N. Engl. J. Med. 306:446-450.

6. Kleerekoper, M., E. Peterson, E. Philips, D. Nelson, B. Tilley, and A. M. Parfitt. 1991. Continuous sodium fluoride therapy does not reduce vertebral fracture rate in postmenopausal osteoporosis. Osteoporosis Int. 1:155.

7. Need, A. G., M. Horowitz, A. Bridges, H. A. Morris, and B. E. C. Nordin. 1989. Effects of nondrolone decanoate and anti resorptive therapy on vertebral density in osteoporotic postmenopausal women. Ann. Intern. Med. 149:57-60.

8. Reeve, J., P. J. Meunier, J. A. Parsons, M. Bernat, O. L. M. Bijvoet, P. Courpron, C. Edouard, L. Klenerman, R. M. Neer, J. C. Renier, et al. 1980 Anabolic effect of human PTH on trabecular bone in involutional osteoporosis: a multicenter trial. Br. Med. J. 280:1340.

9. Bradbeer, J. N., M. Arlot, J. Reeve, and P. J. Meunier. 1988. Human parathyroid peptide (hPTH 1-34) treatment increases the mean wall thickness of iliac trabecular packets of new bone in patients with crush fracture osteoporosis. J. Bone Miner. Res. 3:S160.

10. Hesp, R., P. Hulme, D. Williams, and J. Reeve. 1981. The relationship between changes in femoral bone density and calcium balance in patients with involutional osteoporosis treated with human PTH fragment 1-34. Metab. Bone Dis. Relat. Res. 2:331-334.

11. Hesch, R. D., E-F. Rittinghaus, H. M. Harms, and G. Delling. 1989. Die fruhtherapie der osteoporose mit (1-38) parathormon und calcitonin-nasalspray. Med. Klin. 84:488-498.

12. Saville, P. D. 1969. Changes in skeletal mass and fragility with castration in the rat: a model of osteoporosis. J. Am. Geriatr. Soc. 17:155-169.

13. Aitken, J. M., E. Armstrong, and J. B. Armstrong. 1972. Osteoporosis after oophorectomy in the mature female rat and the effect of estrogen and/or progestogen replacement therapy in its prevention. J. Endocrinol. 55:79-87.

14. Wronski, T. J., P. L. Lowry, C. C. Walsh, and L. A. Ignaszewski. 1985. Skeletal alterations in ovariectomized rats. Calcif. Tissue Int. 37:324-328.

15. Wronski, T. J., C. C. Walsh, and L. A. Ignaszewski. 1986. Histologic evidence for osteopenia and increased bone turnover in ovariectomized rats. Bone. 7:119-123.

16. Kalu, D. N. 1991. The ovariectomized rat model of postmenopausal bone loss. Bone Miner. 15:175-192.
17. Frost, H. M., and W. S. S. Jee. 1992. On the rat model of human osteopenias and osteoporosis. Bone Miner. 18:227-236.

18. Cruess, R. L., and K. C. Hong. 1979. The effect of long term estrogen administration on bone metabolism in the female rat. Endocrinology. 104:11881193.

19. Lindquist, B., A. M. Budy, F. C. McLean, and J. L. Howard. 1960. Skeletal metabolism in estrogen-treated rats studied by means of ${ }^{45} \mathrm{Ca}$. Endocrinology. 66:100-111.

20. Takano-Yamamoto, T., and G. A. Rodan. 1990. Direct effects of $17 \beta$ estradiol on trabecular bone in ovariectomized rats. Proc. Natl. Acad. Sci. USA. 87:2172-2176

21. Hayward, M. A., Y. P. Kharode, M. M. Becci, and D. Kowal. 1990. The effect of conjugated equine estrogens on ovariectomy-induced osteopenia in the rat. Agents Actions. 31:152-156.

22. Kalu, D. N., E. Salerno, C. C. Liu, R. Echon, M. Ray, M. Garzapata, and B. W. Hollis. 1991. A comparative study of the actions of tamoxifen, estrogen and progesterone in the ovariectomized rat. Bone Miner. 15:109-124.

23. Tam, C. S., J. N. M. Heersche, T. M. Murray, and J. A. Parsons. 1982. PTH stimulates the bone apposition rate independently of its resorptive action: differential effects of intermittent and continuous administration. Endocrinology. 110:506-512.

24. Gunness-Hey, M., and J. M. Hock. 1984. Increased trabecular bone mass in rats treated with synthetic human PTH. Metab. Bone Dis. Relat. Res. 5:177181.

25. Hock, J. M., J. Hummert, R. Boyce, J. Fonseca, and L. G. Raisz. 1989. Resorption is not essential for the stimulation of bone growth by human PTH 1-34 in rats in vivo. J. Bone Miner. Res. 4:449-458.

26. Kalu, D. N., R. Echon, and B. W. Hollis. 1990. Modulation of ovariectomy-related bone loss by parathyroid hormone in rats. Mech. Ageing Dev 56:49-62.

27. Gunness-Hey, M., I. Gera, J. Fonseca, L. G. Raisz, and J. M. Hock. 1988. 1,25 dihydroxyvitamin $\mathrm{D}_{3}$ alone or in combination with PTH does not increase bone mass in young rats. Calcif. Tissue Int. 43:284-288.

28. Liu, C. C., D. N. Kalu, E. Salerno, R. Echon, B. W. Hollis, and M. Ray. 1991. Preexisting bone loss associated with ovariectomy in rats is reversed by PTH. J. Bone Miner. Res. 6:1071-1080.

29. Hock, J. M., and I. Gera. 1992. Effects of continuous and intermittent administration and inhibition of resorption on the anabolic response of bone to PTH. J. Bone Miner. Res. 7:65-72.

30. Preece, M. A., J. L. H. O'Riordan, D. E. M. Lawson, and E. Kodicek. 1974. A competitive protein-binding assay for 25 -hydroxycholecalciferol and 25 hydroxyergocalciferol in serum. Clin. Chim. Acta. 54:235-242.

31. Reinhardt, T. A., R. L. Horst, J. W. Orf, and B. W. Hollis. 1984. A microassay for 1,25-dihydroxyvitamin D not requiring HPLC: application to clinical studies. J. Clin. Endocrinol. Metab. 58:91-98.

32. Parfitt, A. M., C. L. E. Matthews, A. R. Villaneuva, M. Kleerekoper, B. Frame, and D. S. Rao. 1983. Relationships between surface, volume and thickness of iliac trabecular bone in aging and in osteoporosis. J. Clin. Invest. 72:13961409.

33. Garrahan, N. J., R. W. E. Mellish, and J. E. Compston. 1986. A new method for the two-dimensional analysis of bone structure in human iliac crest bone biopsies. J. Microsc. 142:341-349.

34. Mellish, R. W. E., M. W. Ferguson-Pell, G. V. B. Cochran, R. Lindsay, and D. W. Dempster. 1991. A new method for assessing two-dimensional cancellous bone structure: comparison between iliac crest and lumbar vertebra. J. Bone Miner. Res. 6:689-696.

35. Stock, J. L., J. A. Coderre, and L. E. Mallette. 1985. Effects of a short course of estrogen on mineral metabolism in postmenopausal women. J. Clin. Endocrinol. Metab. 61:595-600.

36. Nordin, B. E. C., M. Peacock, R. G. Crilly, and D. H. Marshall. 1979. Calcium absorption and plasma $1,25-(\mathrm{OH})_{2}$-D levels in postmenopausal osteoporosis. In Vitamin D. Basic Research and its Clinical Application. Walter de Gruyter, New York. 99.

37. Kalu, D. N., C. C. Liu, E. Salerno, B. Hollis, R. Echon, and M. Ray. 1991 Skeletal response of ovariectomized rats to low and high doses of $17 \beta$-estradiol Bone Miner. 14:175-187.

38. Mitlak, B. H., D. C. Williams, H. U. Bryant, D. C. Paul, and R. M. Neer. 1992. Intermittent administration of bovine PTH (1-34) increases serum 1,25-dihydroxyvitamin D concentrations and spinal bone density in senile ( 23 month) rats. J. Bone Miner. Res. 7:479-484.

39. Delmas, P. D., H. W. Wahner, K. G. Mann, and B. L. Riggs. 1983. Assessment of bone turnover in postmenopausal osteoporosis by measurement of serum bone gla-protein. J. Lab. Clin. Med. 102:470-476.

40. Brown, J. P., L. Malaval, M. C. Chapuy, P. D. Delmas, C. Edouard, and P. J. Meunier. 1984. Serum bone gla-protein: a specific marker for bone formation in postmenopausal osteoporosis. Lancet. 1:1091-1093.

41. Ismail, F., S. Epstein, M. D. Fallon, S. B. Thomas, and T. A. Reinhardt. 1988. Serum bone gla protein and the vitamin D endocrine system in the oophorectomized rat. Endocrinology. 1221:624-630.

42. Fiore, C. E., G. Clementi, R. Foti, A. Prato, and D. R. Grimaldi. 1988. 
Effects of ovariectomy and $17 \beta$-estradiol on bone gla protein in growing rats: an indirect evidence for estrogen receptors in bone cells. Exp. Clin. Endocrinol. 92:335-340.

43. Schepmoes, G., E. Breen, T. A. Owen, M. A. Aronow, G. S. Stein, and J. B. Lian. 1991. Influence of dexamethasone on the vitamin D-mediated regulation of osteocalcin gene expression. J. Cell. Biochem. 47:184-196.

44. Wakamatsu, E., and H. A. Sissons. 1969. The cancellous bone of the iliac crest. Calcif. Tissue Int. 4:147-168.

45. Garrahan, N. J., R. W. E. Mellish, S. Vedi, and J. E. Compston. 1987. Measurement of mean trabecular plate thickness by a new computerized method. Bone. 8:227-230.

46. Mellish, R. W. E., N. J. Garrahan, and J. E. Compston. 1989. Age-related changes in trabecular width and spacing in human iliac crest biopsies. Bone Miner. 6:331-338.

47. Vesterby, A., H. J. G. Gundersen, and F. Melsen. 1989. Star volume of marrow space and trabeculae of the first lumbar vertebra: sampling efficiency and biological variation. Bone. 10:7-13.

48. Vogel, M., M. Hahn, P. Caselitz, J. Woggan, M. Pompesius-Kempa, and G. Delling. 1990. Comparison of trabecular bone structure in man today and an ancient population in western Germany. In Bone Histomorphometry. Proceedings of the Fifth International Congress on Bone Histomorphometry. H. Takahashi, editor. Nishimura Co., Niigata, Japan. 220-223.

49. Compston, J. E., R. W. E. Mellish, P. I. Croucher, R. Newcombe, and N. J. Garrahan. 1989. Structural mechanisms of trabecular bone loss in man. Bone Miner. 6:339-350.

50. Hori, M., T. Uzawa, K. Morita, T. Noda, H. Takahashi, and J. Inoue.
1988. Effect of human parathyroid hormone (PTH(1-34)) on experimental osteopenia of rats induced by ovariectomy. Bone Miner. 3:193-199.

51. Tada, K., T. Yamamuro, H. Okumura, R. Kasai, and H. Takahashi. 1990. Restoration of axial and appendicular bone volumes by h-PTH (1-34) in parathyroidectomized and osteopenic rats. Bone. 11:163-169.

52. Horowitz, J. B., J. Kaye, P. J. Conrad, M. E. Katz, and C. A. Janeway. 1986. Autocrine growth inhibition of a cloned line of helper T cells. Proc. Natl. Acad. Sci. USA. 83:1886-1890.

53. Hodsman, A. B., B. M. Steer, L. J. Fraher, and D. J. Drost. 1991. Bone densitometric and histomorphometric responses to sequential human parathyroid hormone (1-38) and salmon calcitonin in osteoporotic patients. Bone Miner. 14:67-83.

54. Slovik, D. M., D. I. Rosenthal, S. H. Doppelt, J. T. Potts, M. A. Daly, J. A. Campbell, and R. M. Neer. 1986. Restoration of spinal bone in osteoporotic men by treatment with human parathyroid hormone (1-34) and 1,25-dihydroxyvitamin D. J. Bone Miner. Res. 1:377-381.

55. Reeve, J., U. M. Davies, R. Hesp, E. McNally, and D. Katz. 1990. Treatment of osteoporosis with human parathyroid peptide and observations on effect of sodium fluoride. Br. Med. J. 301:314-318.

56. Mosekilde, L., C. H. Sogaard, C. C. Danielsen, O. Torring, and M. H. L. Nilsson. 1991. The anabolic effects of human PTH on rat vertebral body mass are also reflected in the quality of bone, assessed by biomechanical testing: a comparison study between hPTH (1-34) and hPTH (1-84). Endocrinology. 129:421-428.

57. Wronski, T. J., C. F. Yen, and K. S. Scott. 1991. Estrogen and diphosphonate treatment provide long-term protection against osteopenia in ovariectomized rats. J. Bone Miner. Res. 6:387-394. 